\title{
Trends in breeding oat for nutritional grain quality - An overview
}

\author{
Asima Gazal ${ }^{1 *}$, Z. A. Dar², Gul Zaffar ${ }^{2}$, A. A. Lone², I. Abidi ${ }^{3}$, Alima Shabir ${ }^{4}$ and Khan ${ }^{1}$, \\ Nida Yousuf ${ }^{1}$

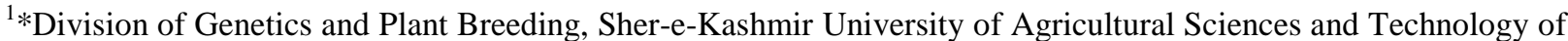 \\ Kashmir, Shalimar-190025 (J\&K), INDIA \\ ${ }^{2}$ Dryland (Karewa) Agricultural Research Station, Budgam, Srinagar- 190001(J\&K), INDIA \\ ${ }^{3}$ Directorate of Research, SKUAST-Kashmir, Shalimar, Srinagar-190025 (J\&K), INDIA \\ ${ }^{4}$ Division of Vegetable Science, SKUAST-Kashmir, Shalimar, Srinagar-190025 (J\&K), INDIA \\ *Corresponding author. E-mail: asimagazal@gmail.com
}

Received: July 13, 2014; Revised received: September 02, 2014; Accepted: November 29, 2014

\begin{abstract}
Oat is an economically important crop and ranks sixth in world cereal production after maize, wheat, rice, barley and sorghum. It has been primarily utilized as livestock feed. However, the utilization of oats for human consumption has increased progressively, owing to its dietary and health benefits which relies mainly on the total dietary fibre and $\beta$-glucan content, which significantly reduces postprandial blood glucose, insulin and blood lipids, especially serum total and low density lipoprotein cholesterol. Henceforth, enhancing Oat b-glucan content for human consumption is desirable. As it is a polygenic trait controlled mainly by genes with additive effects, phenotypic selection for greater b-glucan content would be effective for developing cultivars with elevated b-glucan contents. Oat b-glucan concentration has been found to be positively correlated with protein content and negatively correlated with oil content. $\beta$-glucan yield (i.e., Product of grain yield and $\beta$-glucan content) has been found to correlate positively with both grain yield $(r=0.92)$ and $\beta$-glucan content $(r=0.66)$. Hence, this nutritional oat grain quality has been improved through selection for improved grain yield as they both increase simultaneously. Among wild accessions, A. atlantica genotypes have high $\beta$-glucan content $(2 \cdot 2-11 \cdot 3 \%)$ and have been used in breeding programmes for increasing the $\beta$-glucan content of adapted elite local germplasm. Besides conventional breeding approaches, molecular breeding approaches have made possible to identify several molecular markers linked to $\beta$-glucan rich regions across oat genome hence enabling mapping and dissection of $\beta$-glucan rich genomic regions and accelerating the improvement in nutritional grain quality.
\end{abstract}

Keywords: Avena sativa, $\beta$-Glucan, Breeding, Genetics, Health benefits, Oat grain quality

\section{INTRODUCTION}

Oat (Avena sativa) is the most important cereal fodder crop grown in winter in north western, central India and is now extending to the eastern region. Oat ranks sixth in world cereal production following wheat, maize, rice, barley and sorghum. It is important winter forage in many parts of the world and is grown as multipurpose crop for grain, pasture, forage or as a rotation crop. In India, it is cultivated in Punjab, Haryana, West Bengal, Jammu \& Kashmir, Himachal Pradesh, Uttar Pradesh, Madhya Pradesh, Rajasthan and Maharashtra. The total area covered under oat cultivation in the country is about 5 lakh ha. The crop occupies maximum area in Uttar Pradesh (34\%), followed by Punjab (20\%), Bihar (16\%), Haryana (9\%) and Madhya Pradesh (6\%) (Pandey and Roy, 2011).

Oat is a self-pollinated plant from family Poaceae, native to Europe, Asia and Northwest Africa. It is a natural allopolyploid containing $\mathrm{A}, \mathrm{C}$ and $\mathrm{D}$ genomes formed by the cross between $\mathrm{A}$. barbata and $\mathrm{A}$. strigosa. The ploidy levels include diploid $(2 n=2 x=14)$, tetraploid $(2 \mathrm{n}=4 \mathrm{x}=28)$ and hexaploid $(2 \mathrm{n}=6 \mathrm{x}=42)$ levels. Species grown as crops of minor or regional importance are $A$. byzantina (Cultivated red oat) $(2 \mathrm{n}=6 \mathrm{x}=42$. AACCDD), A. abyssinica (Ethiopian Oat) $(2 \mathrm{n}=4 \mathrm{x}=28, \mathrm{AABB})$, A. strigosa (Lopsided or Bristle or Sand or Black Oat $)(2 \mathrm{n}=2 \mathrm{x}=14$, AsAs $)$ and $A$. nuda (Naked Oat or Hulless Oat) $(2 \mathrm{n}=2 \mathrm{x}=14$, AsAs). Wild species, called as oat-grasses include $A$. barbata (slender oat), A. fatua (wild oat or common wild oat), A. sterilis (winter wild oat or wild red oat), A. brevis (short oat), A. maroccana (Moroccan oat), A. occidentalis (western oat), A. pubescens (downy oat-grass), A. pratensis (meadow oat-grass) and $A$. spicata (poverty oat-grass) (Germeier, 2008). As oats have a high fat, protein and mineral content it was used for medicinal purposes. With the development in field of nutrition, oat was recognized as a healthy food in the mid 1980s signifying that a substance in it helped prevent heart disease and therefore it became more popular for |human nutrition. 
Also oat is a staple in Germany, Ireland, Scotland, and the Scandinavian countries (Butt et al., 2008).

Quality traits of oat grain: Some of the desirable traits required for an oat variety to be bred for human consumption and a variety bred for animal consumption are listed in the table-1 below. Many of the physical characteristics of milling and feed oats are similar, large differences exist in chemical characteristics. Oats used for human consumption has a breeding target of high $\beta$-glucan while as feed oats has a breeding target for lower levels of $\beta$-glucan (Winfield et al., 2007) (Table 1).

Oat $\beta$ Glucan: $\beta$-glucan is a cluster of soluble fiber derived from multiple sources, including cereals (oats and barley), mushrooms, yeast, algae, phyto-plankton, fungi and gram negative bacteria. Each source has different shape and linkage as, cereals have $\beta-(1 \rightarrow 3)$, $(1 \rightarrow 4) \mathrm{D}$ glucan, Lichens have $\beta-(1 \rightarrow 3),(1 \rightarrow 4) \mathrm{D}$ glucan, Yeast have $\beta-(1 \rightarrow 3),(1 \rightarrow 6) D$ glucan and Algae, laminarin have $\beta-(1 \rightarrow 3),(1 \rightarrow 6) D$ glucan. $\beta$-glucan in oat is distributed through the endosperm and is located in the endosperm cell walls constituting about $75 \%$ of the endosperm cell walls shown in figure 1. It is also present in the aleurone cell wall lesser than in endosperm (Miller et al., 1995). Figure 1 shows $\beta$-glucan content in the seed and along the aluerone layer of both the check variety and high $\beta$-glucan mutant variety (Olsson, 2011).

Chemistry of oat b-glucan: Oat b-glucans are non starch polysaccharides. Like starch, they are composed of long chains glucose molecules, but the binding between glucose monomers differs from starch. In starch, the glucose monomers are connected by $\alpha$-bonds viz., amylose has $\alpha$ - $(1 \rightarrow 4)$ bonds, whereas amylopectin, in addition to long glucose chains with $\alpha-(1 \rightarrow 4)$ bonds, also has $\alpha-(1 \rightarrow 6)$ bonds that initiate side chains. The two $\alpha$-bonds in starch are easily digested by enzymes in the intestine. Cellulose is also a polysaccharide and is composed of long chains of glucose molecules bound by $\beta-(1 \rightarrow 4)$, which produces a straight molecule. The intestine does not contain enzymes that can digest these $\beta-(1 \rightarrow 4)$ links. Oat b-glucans, like cellulose, are linear glucose polymers, but oat $\beta$-glucans have both $\beta$ - $(1 \rightarrow 4)$ and $\beta$ - $(1 \rightarrow 3)$ links, creating a cellulose chain with $\beta-(1 \rightarrow 4)$ links interrupted by $\beta-(1 \rightarrow 3)-$ linked glucose units as shown in figure 2. Approximately $70 \%$ of the links are $\beta-(1 \rightarrow 4)$, and the rest are $\beta-(1 \rightarrow 3)$. The distribution is not random, the $(1 \rightarrow 3)$ linkages always occur singly, and most of the $(1 \rightarrow 4)$ linkages occur in groups of three or four. The intestine does not contain enzymes that can digest oat $\beta$-glucans, so they are by definition a fiber (Wood, 1993).

Benefits from $\beta$ - glucan: $\beta$ - glucan is an immunomodulator, it binds to surface receptors and thus causes activation of macrophages, white blood corpuscles (WBC), phygocytosis of neutrophil and activation of lymphocytes thus stimulating antitumour and antimicrobial activity. $\beta$-glucan's immune functions activate the langerhans cells which induces cytokine, particularly of interleukin-1 (il-1) and that stimulates the proliferation of fibro-blasts (skin cells) and synthesis of collagen, elastin and proteoglycan. Therefore, it is used in cosmetics too because it acts as an anti-aging, skin soothing agent, film forming substance, moisturizing and U-VA protectant. Viscosity related properties of $\beta$-glucan can be used in the bakery industry in products such as biscuits and pastas. Its use could also include frozen desserts, breakfast foods, beverages, meats, non-dairy creamers, and canned soups, especially as fat replacer. The cheese industry can benefit from $\beta$-glucans which optimize the process of raw material and improves the cheese structure. The combination of inuline and $\beta$-glucan also has very interesting properties that can be used as fat-replacers in products such as low-fat ice creams. This has been proved to be very successful (UBIC, 2010).

HEALTH BENEFITS OF OATS: Just as we enter our thirties and forties every one among two is suffering from hypertension, high cholesterol, diabetes and many other disorders. Having oats, a whole grain food, which is one of the richest dietary sources of the soluble fibre $\beta$-glucan as a part of our daily diet can help reduce these disorders as it has various $\beta$-glucan health related benefits.

Blood cholesterol and oat $\beta$-glucans: The hypo-cholesterolemic properties of oats were first demonstrated in 1963. Oats were reported to be significantly hypo-cholesterolemic, lowering total and (low density lipoprotein) LDL-cholesterol by 2 to $23 \%$ and they further improve lipid profiles by increasing blood concentrations of high density lipoprotein (HDL) cholesterol as well as apolipoprotein A-I, a major component of HDL (Ripsin et al., 1992; Glore et al., 1994)._Infact, the health benefits of oat rely mainly on the total dietary fibre and $\beta$-glucan content, which lowers blood lipids, especially serum total and LDL -cholesterol (Kerckhoffs et al., 2003). LDL cholesterol, which contains the highest concentration of cholesterol, damages blood vessels because of its tendency to infiltrate and accumulate within arterial walls. LDL-cholesterol, and especially small, dense LDL-cholesterol, is also more susceptible to structural modifications including oxidation and glycosylation, which play major roles in the development of atherosclerosis. High concentrations of HDL are protective against Coronary heart diseases (CHD), since HDL may scavenge and remove excess cholesterol in the arterial wall and also protect LDL against oxidation.

Mode of action: The viscous oat $\beta$-glucans encapsulate bile acids, resulting in their excretion in the faeces. Bile acids generally are recycled, i.e., they are taken up in the lower part of the intestine and used again. Through excretion in faeces, the body loses bile 
acids and has to synthesize new ones, which is done in the liver. The building block for bile acids is cholesterol, which the liver extracts from the blood, decreasing blood cholesterol levels (Marlett et al., 1994).

Fermentation of soluble fiber by bacteria in the large intestine produces propionate (Lia, 1997). The propionate is then absorbed by the colon cells and goes to the liver, where it is thought to have an effect on cholesterol synthesis

Diabetes and oat $\beta$-glucans: Foods rich in carbohydrates have high glycemic response or glycemic index (GI) which cause rapid secretion of insulin from pancreas. Low GI- diet prevents diabetes, cardiovascular disease, metabolic syndrome and obesity. Water-soluble oat $\beta$-glucans exert their effects mainly by increasing viscosity in the small intestine during digestion, resulting in an extended digestion period. Delayed digestion increases blood sugar slowly, causing a low insulin response. The oat $\beta$-glucans forms a protective layer along the intestinal wall that acts as a viscous barrier, slowing food uptake from the intestine (Jenkins et al., 2002; Mckeown et al., 2004).

Blood pressure and oat $\beta$-glucans: High blood pressure (BP) is defined as having a systolic BP greater than $140 \mathrm{~mm} \mathrm{Hg}$ or a diastolic BP greater than $90 \mathrm{~mm} \mathrm{Hg}$. Due to the increasing internal pressure on the artery, hypertension contributes to endothelial injury and increases CHD risk by enhancing the infiltration of LDL particles. Hypertension also a ccelerates the transformation of fatty streaks into fibrous plaques by enhancing smooth muscle proliferation. Oats may help maintain healthy blood pressure by improving the glycemic and insulinemic profiles. The DASH (Dietary Approaches to Stop Hypertension) study demonstrated that a diet high in whole grains, fruit, vegetables and low fat dairy, and restricted in fat, lowers BP in hypertensive individuals. Dietary consumption of oats is consistent with the DASH recommendations, and may confer a benefit due to its fiber content (Harsah et al., 1999; Contreras et al., 2000).

Oat $\beta$-glucans as prebiotics: The lower part of the intestine, the colon, has been identified as a key organ affecting general health. The growth and metabolism of the many individual bacterial species inhabiting the colon depend primarily on the substrates available to them, most of which come from the diet. Oat $\beta$-glucans, which are indigestible in the small intestine but are fermented by bacteria in the colon, are prebiotics. Prebiotics are nondigestible food ingredients that selectively stimulate the growth or activities of bacteria in the colon. They beneficially affect a series of intestinal functions by modulating the structure, composition, and metabolic activity of mucosa and micro flora in the colon. The end products created from prebiotic fermentation in the colon are short chain fatty acids, e.g., butyric acid, that serve as nutrients for mucosal cells (Malkki and Virtanen, 2001).

Oat $\beta$-glucans and weight management: Satiety is a sensation which signals that the stomach is full and it is time to stop eating. When consumed 20 to 30 minutes before eating a meal, oat $\beta$-glucans form a thick viscous fluid in the stomach and small intestine that stimulates the sensation of satiety and helps limit appetite. By reducing the desire for food intake, the effect can help in weight control when combined with a healthy, balanced diet and adequate exercise. As a result of the extended period of digestion, nutrients are utilized by the body over a longer period and, thus, may contribute to a longer period of satiety in weight management programs. Fiber may impart a textural quality that increases chewing time. Fibers have also been reported to prolong gastric emptying, small bowel transit time, and the digestion and absorption of carbohydrates and fat. These actions, which effectively alter glycemic response, have been shown to intensify satiety and aid in the control of energy intake. Meals enriched with beta-glucan elevate plasma levels of cholecystokinin (CCK), a hormone that mediates fat-induced satiety. Fiber may also increase faecal energy excretion. Oat protein has also been found to be more satiating than isoenergetic amounts of carbohydrate or fat (Ludwig, 2000).

Oat and celiac disease: Celiac disease is an autoimmune hereditary disorder of the small intestine that occurs because of sensitivity to gluten in food. Normally the lining of the small intestine has a fluffy velvety texture, but in celiac disorder it becomes smooth and flat. This reduces its ability to absorb nutrients, including sugars, proteins, vital minerals and vitamins from food. When persons with celiac disease take foods containing gluten, their immune system responds by damaging the small intestine lining. Tiny finger like protrusions, called villi are attacked by the immune system and are eventually destroyed. Malnutrition occurs without these villi, no matter how much food a person consumes because the nutrients from food pass the gut without being absorbed (mal absorption), leading to diarrhoea, vitamin and mineral deficiencies, anaemia, osteoporosis and intestinal cancer. Presently, the only effective treatment of celiac disease is a prolonged gluten-free diet. The injurious constituent of wheat in patients with celiac disease is a $\alpha$-gliadin in the prolamin fraction of wheat gluten. Oats do not contain gliadin and its counterpart is avenin. Oats improve the nutritional value of the gluten-free diet without any negative effects on nutritional status and are appreciated by the patients. Inclusion of oats in the gluten free diet is advantageous, since oats are a good source of dietary fiber and of several vitamins and minerals (Huttner and Arendt, 2010).

Additional benefits of oat: In addition to a high content of both soluble and insoluble fiber, oats 
contain lignans, a type of phytoestrogen. They have vasoprotective effects and may reduce the risk for dyslipidemia, hypertension, diabetes, and obesity. Oats provide a unique blend of antioxidants which include wax alcohol, acid esters, avenanthramides and oat saponins. These oat extracts have been shown to inhibit the formation of reactive oxygen species in vitro, which oxidize and promote the atherogenicity of LDL-cholesterol. Compared to other cereal grains, oats contain higher fatty acid content, comprised primarily of linoleic, oleic and palmitic fatty acids. This lipid profile, which contains a higher proportion of polyunsaturated fatty acids (linoleic) and monounsaturated fatty acids (oleic), may also contribute to the hypocholesterolemic and hypotensive effects of oats. Oats also possess an amino acid composition (low lysine to arginine ratio) which may also be cardioprotective and hypocholesterolemic in their effects (Handelman et al., 1999; Anderson et al., 2000).

Oat health claim: A health claim is a statement on a food label which links a food substance to a disease. Few claims are discussed below (Duss, 2005):

Generic oat health claims:

Approved health claims in the United States. Food and drug administration (FDA) 1997.

Soluble fiber from foods such as oat bran, rolled oat, whole oat flour as part of a diet low in saturated fat and cholesterol, may reduce the risk of coronary heart disease. The claim is based on a daily intake of $3 \mathrm{~g}$ of oat $\beta$-glucan and the food product must contain at least 0.75 g per serving.

Approved health claims in Sweden. Voluntary code of practice (SNF) Swedish nutrition, Swedish food federation, Swedish food retailers federation) 2001.

A nutritionally balanced diet rich in oat fibre ( $\beta$-glucan) can reduce the risk of cardiovascular disease or atherosclerosis and must contain $0.75 \mathrm{~g}$ of $\beta$ -glucans per normal serving, or provide $3 \mathrm{~g} \mathrm{day}^{-1}$.

Approved health claims in the United Kingdom. (Joint health claims initiative UK) 2004.

The inclusion of oats as part of a diet low in saturated fat and a healthy lifestyle can help reduce blood cholesterol. Claims relate to oat bran: $5.5 \% \beta$-glucan soluble fibre, rolled oats: $4 \% \beta$-glucan soluble fibre, whole oat flour: $4 \% \beta$-glucan soluble fibre. Daily intake: $3 \mathrm{~g} \mathrm{day}^{-1}$ suggested.

Product specific oat health claims:

PróFIT ${ }^{\circledR}$ bread (4 slices, providing 3 g $\beta$-glucan day- ${ }^{1}$ from OatWell ${ }^{\circledR}$ oat bran). For lowering cholesterol levels in blood (made in Holland).

Primaliv ${ }^{\circledR}$ yogurt [Yogurt $(200 \mathrm{~g})$ provides $4 \mathrm{~g} \beta$-glucan from OatWell® oat bran]. For lowering blood glucose levels, for diabetes (made in Sweden).

\section{GENETICS OF OAT $\beta$ - GLUCAN}

b-glucan content in oat is a polygenic trait controlled mainly by genes with additive effects and no intergenic interaction (Kibite and Edney, 1998). Heritability estimates for $\beta$-glucan content range from 0.27 to 0.58 . (Cervantes-Martinez et al., 2001). $\beta$-Glucan content is less affected by environmental factors, including soil nitrogen level and precipitation (Humphreys et al., 1994). Although genotype environment interaction sometimes is a significant source of variation for $\beta$-glucan content, the ranking of genotypes is generally consistent over environments (Peterson et al., 1995).

\section{BREEDING METHODOLOGIES FOR OAT B- GLUCAN}

Selection: Genotypes having high $\beta$-glucan content are selected and bulked from the source population. Replicated trails for quality and other characteristics are carried and superior ones are carried forward. Genotypes with high $\beta$-glucan are then released. Selection for greater b-glucan content improves grain yield simultaneously. $\beta$-Glucan yield (product of grain yield and $\beta$-glucan content) is positively correlated with both grain yield (with correlation coefficient $(r)=0.92$ ) and $\beta$-glucan content $(\mathrm{r}=0.66)$ (Cervantes-Martinez et al., 2001). b-glucan concentration is found to be positively correlated with protein content. b-glucan concentration is found to be negatively correlated with oil content (Kibite and Edney, 1998). Phenotypic selection for greater groat b-glucan content will be effective for developing cultivars with elevated b-glucan contents. b-glucan concentration is amenable to early generation selection (Cervantes-Martinez et al., 2001) Early generation selection by determination of $\beta$-glucan content is done by following methods:

Enzymatic method: The most accurate and commonly preferred method for $\beta$-glucan analysis is an enzymatic method, based on a bacterial enzyme $[(1-3,1-4)-\beta$-D-glucan 4 glucanohydrolase] produced by Bacillus subtilis. Powder from single seed can be assayed with this method. This method measures soluble $\beta$-glucan content of seeds. For example, content of $\beta$-glucans in oats was found $1.73 \mathrm{~g}$ to $5.7 \mathrm{~g}\left(100 \mathrm{~g}^{-1}\right.$ dry weight) by Havrlentova and Kraic (2006). Other methods include: Micro enzymatic method: A 96 well plate and laser microplate reader that measures the light absorbance in each plate that reflects the amount of $\beta$-glucan in the sample.

Near infrared reflectance spectroscopy (NIRS): This method is cheap, fast (less than 1 min sample ${ }^{-1}$ ) and several components determined at same time but its establishing cost limits its applicability.

Automated flow injection analysis (FIA): The method is based on measurement of intensity of fluorescent absorption of a dye $\beta$-glucan complex. FIA procedure gives consistent sample readings but is costlier method.

Calcoflour flow injection analysis (FIA): Specific binding of calcoflour to $\beta$-glucan results in increased fluorescence intensity which is directly proportional to the concentration. But this method is not for low 
Table 1. Breeding oat gain quality traits for human and animal consumption.

\begin{tabular}{lcc}
\hline Trait & $\begin{array}{c}\text { Breeding target for human } \\
\text { consumption (milling) }\end{array}$ & $\begin{array}{c}\text { Breeding target for animal } \\
\text { consumption (feed) }\end{array}$ \\
\hline Oil per cent & Low & High \\
$\boldsymbol{\beta}$ - glucan (Unique) & High & Low \\
Grain protein per cent & High & High \\
Starch gelantinisation & High & - \\
Hectolitre weight & High & High \\
Taste & Not bitter & - \\
Groat per cent & High & High \\
Grain plumpness & High & High \\
Ease of dehulling & High & High \\
Hull lignin per cent & - & low \\
Grain hardness & High & - \\
Grain colour, brightness & High & High \\
\hline
\end{tabular}

molecular weight $\beta$-glucans.

Pedigree method: Genotypes having high $\beta$-content are crossed and from $\mathrm{F}_{2}$ generation individual plant selection is continued till the progenies become virtually homozygous and show no segregation for $\beta$-glucan content. Selection is done among the progenies because there would be no genetic variation within the progenies. At each stage a record of parent-offspring is kept that is why the method is referred to as pedigree selection. Seeds from superior genotypes are bulked and then released for multi-location testing, licensing, seed multiplication and cultivar release.

Backcross method: Genotypes globally identified as donors for high $\beta$-glucan content are used in back cross programmes eg, Marion has high $\beta$-glucan content (Kianian et al., 2000). HiFi oats which are having about $50 \%$ greater $\beta$-glucan content than other oats (bred by USDA and NDSU) are also used in back cross programmes to increase the $\beta$-glucan content of other local adaptable elite cultivars. In each backcross generation discard $\sim 50 \%$ of first back cross generation $\left(\mathrm{BC}_{1}\right)$ and visually select $\mathrm{BC}_{1}$ progeny that resemble recurrent parent along with having high quality. Process repeated until $\mathrm{BC}_{6}$ so that recurrent parent genome is recovered. Additional back crosses are used till the linkage drag is minimized. If conditions allow marker assisted backcross breeding (MABB) can be used because MABB allows: efficient and accurate selection of target loci of gene of interest, reduces linkage drag and accelerating recovery of recurrent parent thus enabling effective use of resources.

Single seed descent method (SSD): Genotypes having high $\beta$-glucan content are crossed in a crossing block and in $F_{2}$ generation seed from each single plant is bulked and grown for next generation. Process is carried till $\mathrm{F}_{5}$ or $\mathrm{F}_{6}$ till the individual plant progenies become completely homozygous and are referred to as recombinant inbred lines (RILs). In each year, two-three generations may be raised in off season nurseries or mostly in greenhouses. Thus, this method
(SSD) saves time, resources, and rapidly advances homozygosity. Each $\mathrm{F}_{2}$ plant which is a unique combination of two diverse parents is carried till the end population. But the disadvantage of this method is that the size of the end population is very small and no selection is carried till the plants become completely homozygous so the variation is carried as such. After $\mathrm{F}_{5}$ or $\mathrm{F}_{6}$ selection among progenies is done and then the superior ones are evaluated for quality and other characteristics such as preliminary yield trials (PYTs) and coordinated yield trials (CYTs) are done and best genotypes released for seed multiplication.

Use of wild species in breeding programmes: For cultivated A. sativa, groat $\beta$-glucan content usually ranges from 1.8 - 6\% (Cervantes-Martinez et al., $2001)$. Groat $\beta$-glucan concentration showed very wide variation in A. atlantica, A. damascena and A. murphyi genotypes $(2.2$ to $11.3 \%)$. The highest $\beta$-glucan concentrations were found in genotypes of $A$. atlantica (Welch et al., 2000). Henceforth, A. atlantica genotypes may be useful source for increasing the $\beta$-glucan content of cultivated oats. Complete and regular chromosome pairing affinities of interspecific hybrids involving the newly discovered species A. atlantica with $A$. sativa are described due to the AsAs genomic group.

Molecular marker assisted selection breeding (MAS): MAS is much precise and easier as compared to conventional plant breeding. In MAS breeding, tightly linked markers to the target loci or gene of interest are utilized. Therefore, there is an effective and accurate selection of genotypes having target loci or gene of interest. By this method genotypes can be selected at the seedling stage and therefore, there is effective usage of resources. In conventional plant breeding phenotypic selection takes place in field trials and glasshouse trials for many generations which is time consuming and is less effective for accurate selection of genotypes having the gene of interest or target loci. In MAS breeding the only limitation is of 


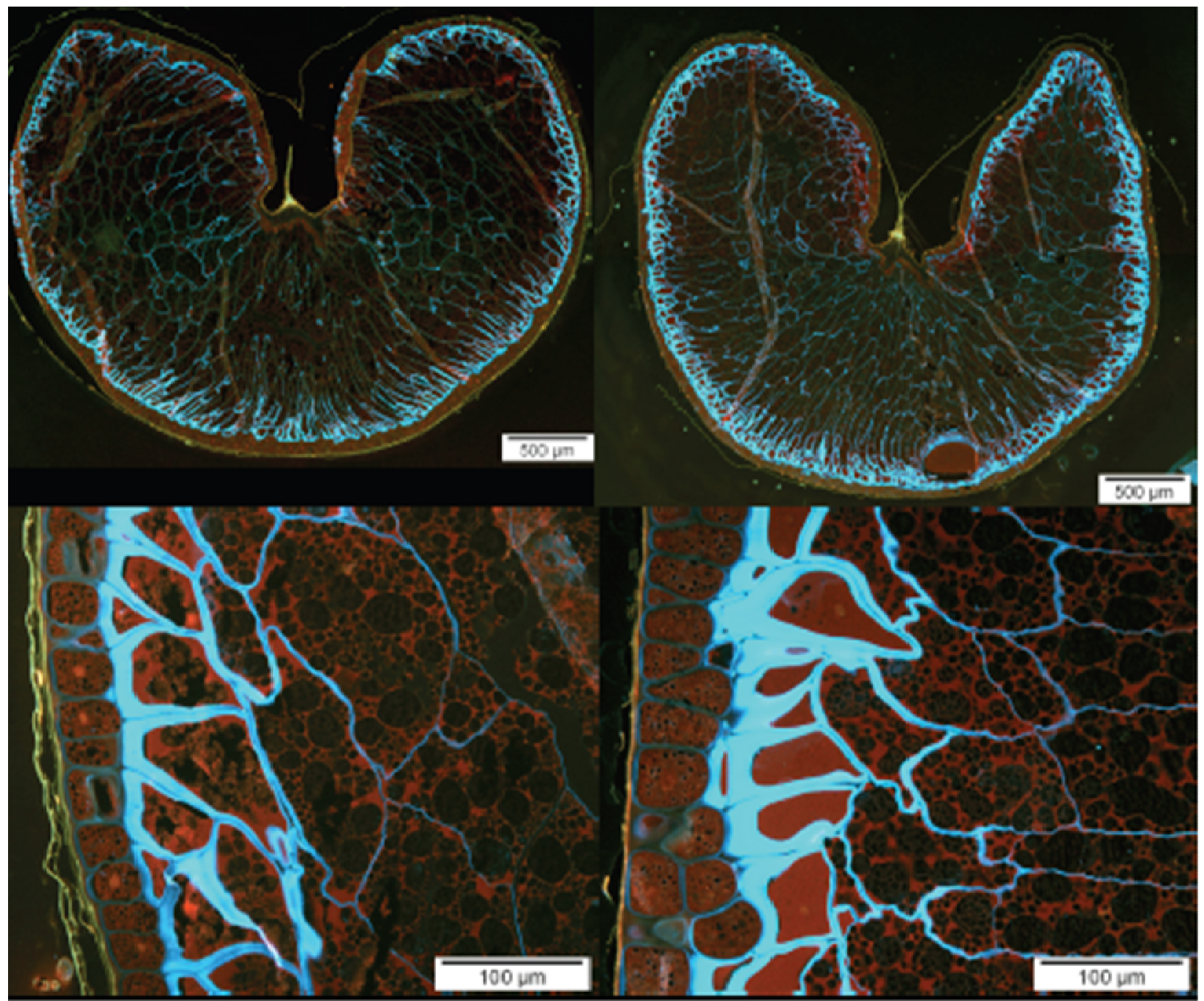

Fig. 1. Localization of $\beta$-glucan in oat seed kernels visualized by: Chalcoflour staining of seed cross sections.. Calcofluor-stained $\beta$-glucan appears blue. (Top Left) Seed cross-section of the commercial cultivar SW Belinda. (Check) (Top Right) Seed cross-section of the high $\beta$-glucan mutant CT828. (Bottom Left) Magnified view of the aleurone layer in SW Belinda. (Bottom Right) Magnified view of the aleurone layer in CT828.

high cost.

Quantitative trait loci (QTL) mapping: Compared to other crops like wheat, rice and barley, mapping of the oat genome lagged behind. This was partly due to the large genome size of oat and its polyploid nature. Particularly, unlike wheat, oat lacked a complete set of either nullisomic or nullitetrasomic lines, which makes it difficult to localize molecular marker loci to specific chromosomes. First molecular linkage map was developed by O'Donoughue et al. (1995), using (RIL) population from a cross between $A$. byzantina C. Koch cv Kanota and A. sativa L. cv Ogle (Groh et al., 2001 and Wight et al., 2003). The first QTL analysis for oat $\beta$-glucan content was conducted by Kianian et al. (2000). Two recombinant inbred populations sharing a common parent (Kanota x Ogle (KO) and Kanota $\mathrm{x}$ Marion (KM)) were used to identify genomic regions that influence $\beta$ glucan content in cultivated oat. Regions influencing $\beta$-glucan content on linkage groups 11 and 14 of the oat KO RFLP map are identified in both populations and over environments. Genetic analysis of $\beta$-glucan content in $\mathrm{KO}$ identified 8 QTLs on the 7 LGs (KO3+38, KO6, KO11-41+20, KO13, KO14, KO17 and KO19+27). KM map includes regions homologous to the $\mathrm{KO} \beta$-glucan content QTL regions and five genomic regions that affect $\beta$-glucan content are identified (on KM7, KM11, KM14, KM5X and KM10X) (Kianian et al., 2000 and Groh et al., 2001). Molecular marker recombination maps for Terra $\mathrm{x}$ Marion (TM) have also been developed five genomic regions are |identified in TM population associated with $\beta$ - glucan (TM5, TM18, TM 21, TM29 and one unlinked AFLP marker) (De Koeyer et al., 2004). Many markers have been mapped to $\beta$-Glucan QTL regions in Oat. Total 18 markers have been identified that are tightly linked to the target loci $(<5 \mathrm{cM})$ (Orr and Molnar, 2008).

An alternative approach for QTL detection are genome -wide association studies (GWAS). This approach is also dependent on the level of Linkage disequilibrium 


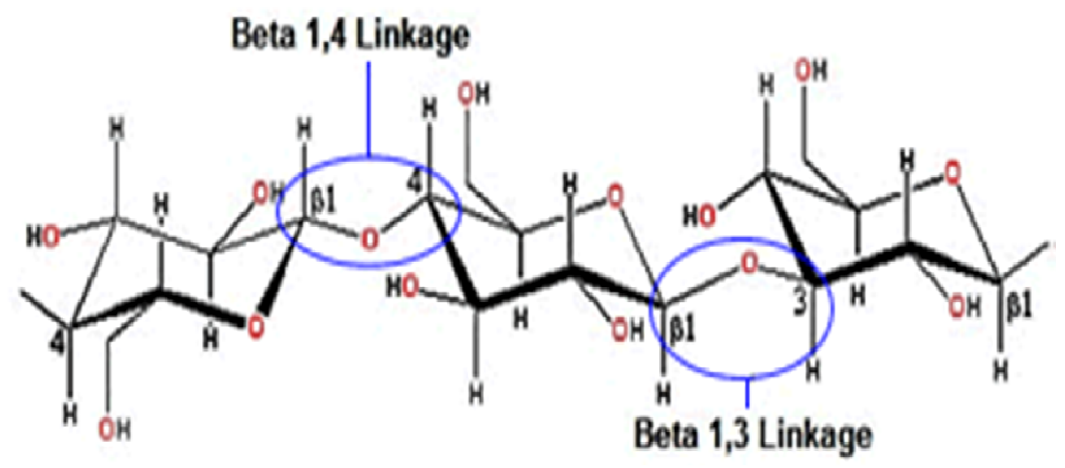

Fig. 2. Typical oat soured $\beta$-glucan shape.

(LD) ie., the non random association of alleles at different loci, but, unlike linkage mapping, it depends on historical LD generated prior to any experimental work and broken down by many generations of recombination. Thus, chromosomal regions that remain in LD span shorter distances and have the potential to deliver high-resolution mapping. Additionally, because GWAS utilizes populations of lines unrelated by any specific crossing design (Zhu et al., 2008); the allelic diversity sampled is likely to be greater than two alleles per locus as is the case for linkage mapping. The resolution for GWAS is not only dependent on the extent of LD but also the marker density. Recent advances in marker technology for oat have led to the development of Diversity Array Technology (DArT) markers (Tinker et al., 2009). DArT development, have been utilized in genomic selection for oat $\beta$-glucan and yield (Asoro et al., 2011). The marker density that has been gained with development of the DArT marker system also enables the application of GWAS to detect QTL controlling $\beta$-glucan content in oat. Identification of QTL controlling $\beta$-glucan content has been limited in oat (Kianian et al., 2000; De Koeyer et al., 2004). Fortunately, a comparative genomics approach can give some insight into candidate genes from other grass species (Fincher, 2009). First identified in barley (Han et al., 1995) and later identified on chromosome seven of rice (Burton et al., 2006), the CslF gene family is known to have $\beta$-glucan synthase function. Thus, this gene family is an important candidate for QTL detection for increased $\beta$-glucan content in oat.

Other approaches

Advanced backcross - Quantitative trait loci (AB-QTL) analysis: It is a directed approach to introduce QTLs for b-glucan content from a gene bank accession (wild species) into elite cultivar breeding programmes (Tanksley and Nelson, 1996). In a case study, segregation for $\beta$-glucan content was evident from three $\mathrm{BC}_{2} \mathrm{~F}_{1}$ populations consisting of 98,73 and 67 lines from "Iltis $\mathrm{x}$ IAH611-447(high $\beta$-glucan donor)" (Jianzhong et al., 2004). To overcome the drawbacks in conventional QTL analysis, Tanksley and Nelson (1996) reported a novel strategy: advanced backcross QTL analysis. They combined the mapping of interested QTL alleles and the introgression of these favourable alleles from exotic germplasms into one process by delaying QTL analysis until an advanced generation like $\mathrm{BC}_{2}$ or $\mathrm{BC}_{3}$. There are several advantages utilizing this method for QTL analysis as outlined by Tanksley and Nelson (1996):

Phenotypic selections can be conducted in early generations hence reducing the frequency of undesirable alleles from the donor and major negative QTLs.

The advanced generation is skewed towards alleles from the recurrent parent, reducing the possibility for QTL detection with epistatic interactions, but makes it easier to detect additive QTLs which will still function in the nearly isogenic background of the recurrent parent.

The mean performance of the advanced generation is skewed towards the elite parent, making subtle pleiotropic effects easier to be detected.

Only few additional backcross generations will be needed to create nearly isogenic lines with selected QTLs. Such QTL-NILs (Quantitative Trait Loci- Near Isogenic Lines) will be potential candidates for new varieties with enhanced attributes.

TILLING (Targeting Induced Local Lesions IN Genomes): This method combines high frequency mutagenesis (with a chemical mutagen viz., Ethyl Methane Sulfonate) along with molecular based, high precision selection technique that identifies single base mutations in a target gene. TILLING is a good alternative to more direct DNA modifying techniques since seed mutagenesis is easy to apply and relatively independent of genome size and organization (Henikoff and Comai, 2003). In a case study, oat population of 2600 mutagenised M2 lines, producing 2550 M3 seed lots was developed and the mutation frequency determined. Chawade et al., 2010 demonstrated the potential of the oat TILLING population by identifying ten different mutations in the cellulose synthase-like (AsCslF6) $\beta$-glucan biosynthes gene - key gene in the $\beta$-glucan biosynthetic pathway. Since $\beta$-glucans are becoming very important functional food ingredients, an oat variety with increased or modified $\beta$-glucan content in the seed will significantly increase the value of the crop. 
Gene transfers: Gene transfers of microbial 1, 3- $\beta$-glucan synthases (Inoue et al., 1995). In a case study this was accomplished by using particle bombardment (Wan and Lemaux, 1994).

\section{Conclusion}

Oat is considered $100 \%$ whole grain food, as it provides carbohydrates, protein and is one of the richest dietary sources of the soluble fibre $\beta$-glucan. Many health related benefits of oats result from the high viscosity of the soluble $\beta$-glucan. Viscosity is the thickness or resistance to flow of a liquid. $\beta$ - glucan accomplishes its nutritional functions by increasing the viscosity of fluids in the gut. Therefore, it has been proposed as a target trait in oat breeding programs so as to double its concentration. b-glucan is a polygenic trait controlled mainly by additive genes henceforth, phenotypic selection for high b-glucan content is effective for developing cultivars with high b-glucan contents. Oat b-glucan content has been found to be positively correlated with protein content and negatively correlated with oil content. $\beta$-Glucan yield (i.e., Product of grain yield and $\beta$-glucan content) has been found to correlate positively with both grain yield $(r)=0.92)$ and $\beta$-glucan content $(r=0.66)$. Also, heritability estimates for $\beta$-glucan content ranges from 0.27 to 0.58 . Therefore, selection for higher grain yield in oat varieties improves $\beta$-glucan content simultaneously. Several breeding methods like phenotypic selection for greater groat b-glucan content and also early generation selection by determination of $\beta$-glucan content at only seedling stage, pedigree, SSD and back cross are utilized to breed for oat b-glucan content. Wild accessions viz., A. atlantica have highest $\beta$-glucan concentrations $(2 \cdot 2-11 \cdot 3 \%)$ and thus, can be used in breeding programmes for increasing the $\beta$-glucan content of elite, adapted, local germplasm via backcross programmes. Besides, conventional breeding methods, molecular breeding (MB) approaches viz., MAS, MABB, AB-QTL and genome wide association studies (GWA) along with mutagenesis (TILLING) have made possible to identify and dissect $\beta$-glucan content rich regions across oat genome. Hence, such approaches have enabled to map and to dissect these genomic regions, accelerating improvement in oat grain quality and increasing the nutritional value of the crop for human consumption.

\section{REFERENCES}

Anderson, J.W., Hanna, T.J., Peng, X., and Kryscio, R.J. (2000). Whole grain foods and heart disease risk. J. Am. Coll. Nutr., 19: 291-299.

Asoro, F.G., Newell, M.A., Beavis, W.D., Scott, P. and Jannink, J.L. (2011). Accuracy and training population design for genomic selection on quantitative traits in elite North American oats. Plant Genome, 4: 132-144.

Burton, R.A., Wilson, S.M., Hrmova, M., Harvey, A.J.,
Shirley, N.J., Medhurst, A., Stone, B.A., Newbignin, E.J., Bacic, A., and Fincher, G.B. (2006). Cellulose synthase - like CslF genes mediate the synthesis of cell wall $(1,3 ; 1,4)-\beta-\mathrm{d}$ - glucans. Science, 311: 1940-1942.

Butt, M.S., Nadeem, M.T., Khan, M.K.I., Shabir, R. and Butt, M.S. (2008). Oat: unique among the cereals. Eur. J. Nutr., 47: 68-79.

Cervantes-Martinez, C.T., Frey, K.J., White, P.J., Wesenberg, D.M. and Holland, J.B. (2001). Correlated responses to selection for greater $\beta$-glucan content in two oat populations. Crop Science, 42: 1730-1738.

Chawade, A., Sikora, P., Brautigam, M., Larsson, M., Vivekanand, V., Nakash, M.A., Chen, T. and Olsson, O. (2010). Development and characterization of an oat TILLING population and identification of mutations in lignin and $\beta$-glucan biosynthesis genes. BMC Plant Biology, 10: 86-98.

Contreras, F., Rivera, M., Vasquez, J., De la Parte, M.A. and Velasco, M. (2000). Diabetes and hypertension patho physiology and therapeutics. J. Hum. Hyperten., 1: 26-31.

De Koeyer, D.L., Tinker, N.A., Wight, C.P., Deyl, J., Burrows, V.D. and O'Donoughue, L.S. (2004). A molecular linkage map with associated QTLs from a hulless $\mathrm{x}$ covered spring oat population. Theortical Applied Genetics, 108: 1285-1298.

Duss, R. (2005). Oat bran: proven and advanced nutrition for your heart Innovations in Food Technology 19: 122-126.

Fincher, G.B. (2009). Exploring the evolution of $(1,3 ; 1,4)$ - $\beta$-D-glucans in plant cell walls: comparative genomics can help! Curr. Opin. Plant. Biol., 12: 140-147.

Germeier, C.U. (2008). Global strategy for the ex situ conservation of oats (Avena spp.) federal centre for breeding research on cultivated plants (BAZ) Quedlinburg, Germany, pp. 10.

Glore, S.R., Van Treeck, D., Knehans, A.W. and Guild, M. (1994). Soluble fiber and serum lipids: a literature review. Journal of American Diet Association, 94: 425 -436 .

Groh, S., Zacharias, A., Kianian, S.F., Penner, G.A., Chong, J., Rines, H.W. and Phillips, R.L. (2001). AFLP product homology in comparative mapping in two hexaploid oat populations. Theortical and Applied Genetics, 102: 876 -884 .

Han, F., Ullrich, S.E., Chirat, S., Menteur, S., Jestin, L., Sarrafi, A., Hayes, P.M., Jones, B.L., Black, T.K., Wesenberg, D.M., Kleinhofs, A. and Kilian, A. (1995). Mapping of beta-glucan content and beta-glucanase activity loci in barley grain and malt. Theor. Appl. Genet., 91: 921-927.

Handelman, G.J., Cao, G., Walter, M.F., Nightingale, Z.D., Paul, G.L., Prior, R.L. and Blumberg, J.B. (1999). Antioxidant capacity of oat (Avena sativa L.) extracts. 1. Inhibition of low-density lipoprotein oxidation and oxygen radical absorbance capacity. Journal of Agriculture and Food Chemistry, 47: 4888-4893.

Harsah, D.W., Lin, P.H. and Obarzanek, E., Karanja N.M., Moore, T.J. and Caballero, B. (1999). Dietary Approaches to Stop Hypertension: a summary of study results. DASH Collaborative Research Group. Journal of American Diet Association, 8: 35-39.

Havrlentova, M. and Kraic, J. (2006). Content of beta -d-glucan in cereal grains. Journal of Food Research and Nutrition, 45: 97-103.

Henikoff, S. and Comai, L. (2003). Single-nucleotide mutations for plant functional genomics. Annual 
Revolution Plant Biology, 54: 375-401.

Humphreys, D.G., Smith, D.L. and Mather, D.E. (1994). Nitrogen fertilizer and seeding date induced changes in protein, oil, and $\beta$-glucan contents of four oat cultivars. Journal of Cereal Science, 20: 283-290.

Huttner, E.K. and Arendt, E.K. (2010). Recent advances in gluten-free baking and the current status of oats. Trends in Food Science \& Technology, 21: 303-312.

Inoue, S.B., Takewakt, N., Mio, T., Adachi, M., Fujii, Y., Miyamoto, C., Arisawa, M., Furuichi Y. and Watanabe, T. (1995). Characterization and Gene Cloning of $1,3-\beta$ -D-Glucan Synthase from Saccharomyces Cerevisiae. European Journal of Biochemistry, 231: 845 -854 .

Jenkins, A.L., Jenkins, D.J.A., Zdravkovic, U., Wursch, P. and Vuksan, V. (2002). Depression of the glycemic index by high levels of $\beta$-glucan fiber in two functional foods tested in type 2 diabetes. European Journal of Clinical Nutrition, 56(7): 622-628

Jianzhong, Y., Beuch, S., Herrmann, M. and Hackauf, B. (2004). AB- QTL analysis for $\beta$-glucan content in oats. Proceedings $7^{\text {th }}$ International oat conference. Pirjo Peltonen-Sainio and Mari Topi-Hulmi (eds.) MTT Agri food Research Finland pp. 52.

Kerckhoffs, D., Hornstra, G. and Mensink, R. (2003). Cholesterol lowering effect of $\beta$-glucan from oat bran in mildly hypercholesterolemic subjects may decrease when $\beta$-glucan is in corporate into bread and cookies. American Journal of Clinical Nutrition, 78: 221-227.

Kianian, S.F., Phillips, R.L., Rines, H.W., Fulcher, R.G., Webster, F.H. and Stuthman, D.D. (2000). Quantitative trait loci influencing $\beta$-glucan content in oat (Avena sativa, $2 \mathrm{n}=6 \mathrm{x}=42)$. Theortical Applied Genetics, 101: $1039-1048$

Kibite, S. and Edney, M.J. (1998). The inheritance of $\beta$ glucan concentration in three oat (Avena sativa L.) crosses. Canadian Journal of Plant Science, 78: 245 -250 .

Lia, A. (1997). Effects of oat fibre on nutrient absorption and sterol metabolism. Ph.D. thesis. Goteborg University, Goteborg, Sweden.

Ludwig, D.S. (2000). Dietary glycemic index and obesity. American Society of Nutrition Science, 130(2): 280 S $-283 S$.

Malkki, Y. and Virtanen, E. (2001). Gastrointestinal effects of oat bran and oat gum: A review. Lebensm.-Wiss. Technol., 34: 337-347.

Marlett, J.A., Hosig, K.B., Vollendorf, N.W., Shinnick, F.L., Haack, V.S. and Story, J.A. (1994). Mechanism of serum cholesterol reduction by oat bran. Hepatology, 20:1450-1457

McKeown, N.M., Meigs, J.B., Liu, S., Saltzman, E., Wilson, P.W.F. and Jacques, P.F. (2004). Carbohydrate nutrition, insulin resistance, and the prevalence of the metabolic syndrome in the Framingham Offspring Cohort. Diabetes Care, 27(2): 538-546.

Miller, S.S., Fulcher, R.G., Sen, A. and Arnason, J.T. (1995). Oat endosperm cell walls: I. Isolation, composition, and comparison with other tissues. Cereal Chemistry, 72: 421-427.

O’Donoughue, L.S., Kianian, S.F., Rayapati, P.J., Penner, G.A., Sorrells, M.E., Tanksley, S.D., Phillips, R.L.,
Rines, H.W., Lee, M., Fedak, G., Molnar, S.J., Hoffman, D., Salas, C.A., Wu, B., Autrique, E., Van and Deynze, A. (1995). A molecular linkage map of cultivated oat. Genome, 38: 368-380.

Olsson, O. (2011). Molecular breeding for enhanced food and feed quality in oat. Nordic Oat Days, pp: 14-16.

Orr, W. and Molnar, S.J. (2008). Development of PCR based SCAR and CAPS markers linked to glucan and protein content QTL regions in oat. Genome, 51: 421-425.

Pandey, K.C. and Roy, A.K. (2011). Forage Crops Varieties. IGFRI Jhansi (India) pp. 16-17.

Peterson, D.M., Wesenberg, D.M. and Burrup, D.E. (1995). $\beta$-Glucan content and its relationship to agronomic characteristics in elite oat germplasm. Crop Science, 35: 965-970.

Ripsin, C.M., Keenan, J.M., Jacobs, D.R., Elmer, P.J., Welch, R.R., Van Horn, L., Liu, K., Turnbull, W.H., Thye, F.W., Kestin, M., Hegsted, M., Davidson, D.M., Davidson, M.H., Dugan, L.D., Demark-Wahnefried, W. and Beling, S. (1992). Oat products and lipid lowering: a meta-analysis. Journal of American Medical Association, 267: 3317-3325.

Tanksley, S.D. and Nelson, J.C. (1996). Advanced backcross QTL analysis: a method for the simultaneous discovery and transfer of valuable QTLs from unadapted germplasm into elite breeding lines. Theortical and Applied Genetics, 92: 191-203.

Tinker, N.A., Kilian, A., Wight, C.P., Heller-Uszynska, K., Wenzl, P., Rines, H.W., Bjørnstad, A., Howarth, C.J., Jannink, J.L., Anderson, J.M., Rossnagel, B.G., Stuthman, D.D., Sorrells, M.E., Jackson, E.W., Tuvesson, S., Kolb, R.L., Olsson, O., Federizzi, L.C., Carson, M.L., Ohm, H.W., Molnar, S.J., Scoles, G.J., Eckstein, P.E., Bonman, J.M., Ceplitis, A., and Langdon, T. (2009). New DArT markers for oat provide enhanced map coverage and global germplasm characterization. BMC Genomics, 10: 39. (doi: 10.1186/1471-2164-10-39).

UBIC (2010). The world $\beta$ - glucan ingredient market. UBIC - Consulting.

Wan, Y. and Lemaux, P.G. (1994). Generation of Large Numbers of Independently Transformed Fertile Barley Plants. Plant Physiology, 104: 37-48.

Welch, R.W., Brown, J.C.W. and Leggett, J.M. (2000). Interspecific and Intraspecific Variation in Grain and Groat Characteristics of Wild Oat (Avena) Species: Very High Groat $(1 \rightarrow 3),(1 \rightarrow 4)-\beta$-glucan in an Avena atlantica Genotype. Journal of Cereal Science, 31(3): 273-279.

Wight, C.P., Tinker, N.A., Kianian, S.F., Sorrells, M.E., O'Donoughue, L.S. and Hoffman, D.L. (2003). A molecular marker map in 'Kanota' $\mathrm{x}$ 'Ogle' hexaploid oat (Avena spp.) enhanced by additional markers and a robust framework. Genome, 46: 28-47.

Winfield, K., Hall, M. and Paynter, B. (2007). Milling oat and feed oat quality - what are the differences? Department of Agriculture and Food, 4703: 1833-7236.

Wood, P.J. (1993). Physiochemical characteristics and physiological properties of oat $(\lg 3),(1 \mathrm{~g} 4)-\beta$ - D - glucan. In: Oat Bran. P.J. Wood, ed. American Association of Cereal Chemists, St. Paul, MN: 83.

Zhu, C., Gore, M., and Buckler, E.S. (2008). Status and prospects of association mapping in plants. Plant Genome, 1: 2-5. 